\title{
Foraging Swarms as Nash Equilibria of Dynamic Games
}

\author{
Arif Bülent Özgüler and Aykut Yıldız, Student Member, IEEE
}

\begin{abstract}
The question of whether foraging swarms can form as a result of a noncooperative game played by individuals is shown here to have an affirmative answer. A dynamic game played by $N$ agents in 1-D motion is introduced and models, for instance, a foraging ant colony. Each agent controls its velocity to minimize its total work done in a finite time interval. The game is shown to have a unique Nash equilibrium under two different foraging location specifications, and both equilibria display many features of a foraging swarm behavior observed in biological swarms. Explicit expressions are derived for pairwise distances between individuals of the swarm, swarm size, and swarm center location during foraging.
\end{abstract}

Index Terms-Artificial potentials, differential game, Hamilton-Jacobi, multiagent systems, Nash equilibrium, swarm.

\section{INTRODUCTION}

$\mathbf{S}$ WARM MODELING is a research topic that has attracted the attention of many diverse disciplines such as physics, biology, and engineering. Swarming behavior has been the basis for modeling of multirobot systems, multivehicle systems, and also optimization algorithms. This multiagent system modeling is mainly inspired by biological behaviors such as schooling of fish, flocking of birds, and herding of sheep, as stated in [1]. Therefore, swarming behavior was initially studied for the purpose of biological modeling. The term swarming behavior is defined as the cooperative coordination of animals of the same species to achieve aggregation by forming clusters [2]. This behavior has many advantages such as reducing individual efforts, increasing the immigration distances, providing safety of the animals, and also enhancing the foraging performance [3]. For instance, the reasons behind the flocking of birds in $\mathrm{V}$ formation are effort reduction and longer immigration distances [4].

One of the most important applications of swarming is the motion planning of teams of robots. In a multiple robot system, the robots keep a formation while navigating to a target location. In this setting, the agents achieve a cooperative task by exchanging information with the others, while controlling their individual dynamics [5]-[7]. Here, using a team of simple robots instead of one sophisticated robot increases the

Manuscript received September 19, 2012; revised July 8, 2013; accepted September 11, 2013. Date of publication October 7, 2013; date of current version May 13, 2014. This paper was recommended by Associate Editor T. Vasilakos.

The authors are with the Department of Electrical and Electronics Engineering, Bilkent University, Ankara 06800, Turkey (e-mail: ozguler@ee.bilkent.edu.tr; ayildiz@ee.bilkent.edu.tr).

Digital Object Identifier 10.1109/TCYB.2013.2283102 robustness and resilience against communication errors [8]. An example of optimal motion planning for multiple robots is [9].

Another biologically inspired field related to swarms is the coordination of multiple vehicle systems. The swarm theory has been applied to both platooning of vehicles and air traffic control. Conflicts in the intersection crossings have been resolved by swarm theory in [10] and [11] for vehicle platooning on automated highways. In the current air traffic control mechanism, the planes fly in predefined paths, which may deviate from the shortest path significantly. In future free flight paradigm that is discussed in [12] and [13], the air vehicles will arbitrarily select the elevation, speed and path, and the conflicts will be resolved by intelligent collision avoidance algorithms. Such future multivehicle systems, namely the unmanned aerial vehicles, are studied in [14]. Another important application of swarming behavior is optimization. The recent versions of such an algorithm, particle swarm optimization, are [15] and [16].

Artificial potentials are commonly used to model the interaction between individuals in multiagent systems. In this technique, the interaction is modeled as attractions and repulsions between the individuals so that a cluster form is maintained [17], [18]. The individuals repel the neighbors in near field, and attract them in the far field. One of the first works that exploited artificial potentials is [19]. In that work, a set of individuals is selected as virtual leaders so that the system is semidecentralized to achieve scalability. Another work that employs artificial potentials and that includes stability analysis is [20].

Social foraging is defined as the searching act of a group of animals for food or better environment. In [21], the problem of the animal decision making in social foraging is modeled in a game theoretical framework. In that work, the effect of the ratio of the producers and scroungers on foraging performance is investigated. In [22], foraging is modeled as the minimization of a scalar field that represents the toxicity and food characteristics of the environment.

Open-form algorithms are widely used to analyze multiagent systems. However, convergence, accuracy, and computational complexity can be problematic in algorithm-based techniques as opposed to closed-form solutions. An example of a collision avoidance algorithm based on near field repulsion is [23]. Lyapunov-based techniques are also applied [24], [25] and focus on the stability of the system, but do not yield explicit solutions of the dynamics of the system. A method that yields an explicit solution of the system is, of course, 
preferable since it would lead to a simulation with low complexity and display the stability of the system with ease. A resource on obtaining explicit solutions of linear quadratic games is [26].

In this paper, a game theoretical model is introduced to examine how swarms form as in, for instance, the foraging behavior of ant colonies or in platooning of vehicles on automated highways. This is an individual focused study of swarms that questions whether a swarm can form in a time interval by noncooperative actions of a finite number of individuals or agents. Here, we assume that each agent in a group, while in search of, say, food, minimizes its total effort by using the force that it applies as a control input. This leads to an $N$-person infinite-dimensional dynamic game [27], and to the question of whether this game has a Nash equilibrium that carries the features of a swarm. An affirmative answer means that noncooperative optimization by $N$ individuals results in a collective behavior, namely swarming behavior. The answer indeed turns out to be affirmative for particular individual cost functionals into which artificial potential energy [22] terms that represent the tradeoff between repulsion and attraction are incorporated.

Game theory, in particular evolutionary game theory, has been extensively applied to analysis of swarm behavior and animal decision making [21], [28]. The use of game theory in social foraging, such as in [28], is limited to two-person games since the objective is to predict and explain the foraging behaviors of animals while in groups. A combination of game theory and optimal control theory has also been applied to the modeling of dynamic behaviors of multiagent systems such as in [27]. The cooperative control of a multiagent system has been formulated as the Hamilton-Jacobi form in a differential game framework in [29] and [30]. In [31], game theory is employed for the optimal network consensus problem. The dynamic (or differential) game model introduced here is different from the models in these works since it is a noncooperative $N$-person game and focuses on a Nash equilibrium.

Our main contribution is to model foraging swarm behavior as a noncooperative game played by $N$ individuals and to show that the game has a Nash equilibrium that is unique with respect to a class of strategies. This indicates that a swarming behavior can result from noncooperative actions of individuals. The Nash equilibrium solution for this game is described explicitly, i.e., expressions for optimal trajectories, swarm size, and center trajectory are obtained. The game is analyzed under two different terminal conditions.

This paper is organized as follows. Section II is on the problem definition and introduces the dynamic games. In Section III, main results on existence and uniqueness of Nash equilibria are presented. Section IV contains the simulation results and the last section is on conclusions. The proofs of Theorems 1 and 2 and of Corollary 1 in Section III are given in the Appendix. The results of this paper have been partly presented in [32].

\section{PROBLEM DEFINITION}

1-D swarm behavior of $N$ agents, such as the flocking of ants in a queue, will be modeled as a noncooperative
TABLE I

LIST OF NOTATIONS

\begin{tabular}{|l|l|}
\hline Symbol & Description \\
\hline$N$ & Number of agents (players) \\
$L^{i}$ & Cost minimized by $i^{t h}$ player \\
$u^{i}(t)$ & Input applied by $i^{t h}$ player \\
$x^{i}(t)$ & Optimal trajectory of $i^{t h}$ player \\
$T$ & Terminal time \\
$a, r$, and $f$ & Attraction, repulsion, and foraging parameters \\
$\alpha$ & $\sqrt{N a}$ \\
$s^{i}(t)$ & Sums of signs of pairwise relative positions \\
$d(t)$ & Swarm size at time $t$ \\
$t^{*}$ & Time for maximum swarm size \\
$\bar{x}(t)$ & Optimal trajectory of the swarm center \\
$p^{i}(t)$ & Lagrange multiplier of $i^{t h}$ player \\
$\phi(t)$ & State transition matrix \\
\hline
\end{tabular}

infinite-dimensional dynamic game. Two such games that make different assumptions on the foraging target are introduced in this section.

It is assumed that each agent minimizes its individual total effort in a time interval by controlling its velocity. The total work done in a finite interval $[0, T]$ that is minimized by agent$i$ is given by

$$
\begin{aligned}
& L^{i}:=\left[x^{i}(T)\right]^{2} f+\int_{0}^{T}\left\{u^{i}(t)^{2}\right. \\
& \left.+\sum_{j=1, j \neq i}^{N}\left(a\left[x^{i}(t)-x^{j}(t)\right]^{2}-r\left|x^{i}(t)-x^{j}(t)\right|\right)\right\} d t
\end{aligned}
$$

where $f, a$, and $r$ are positive constants. The $T$ parameter is the duration for foraging of the colony, $x^{i}(t)$ is the position of the $i$ th agent at time $t$ and $N$ is the number of agents. The first term penalizes the distance to the foraging location at the final time, which is assumed to be the origin in $x^{1} \ldots x^{N}$-space. This formulation specifies a very simple attractant/repellent profile [22]. The second term in the integrand gives the attraction potential energy, and the last term, the repulsion potential energy. These terms are introduced as a result of the assumption that each agent measures its distance to every other agent and optimizes these distances so as to remain as close as possible to every other agent without getting too close to any one of them. Introduction of such terms into the total potential energy and its (cooperative) minimization have been shown to lead to stable swarms in the stability analysis of [22]. The first term of the integrand in (1) is the contribution to the total work done by agent's kinetic energy. Using velocity as a control input $u^{i}(t)=\dot{x}^{i}(t)$ arises from applying force in a viscous environment at which particle mass is neglected [22]. Thus, each agent minimizes its total effort, total work done, during the foraging process. The weights $a$ and $r$ are introduced in order to compare the relative effects of the attraction and repulsion terms in the integrand. The weight $f$, on the other hand, will allow us to measure the severity of the distance-totarget penalization. Here, we assume that the individuals are identical point agents with the same attraction and repulsion parameters. Moreover, we assume that all of the particles play one foraging swarm game altogether. (If we consider that the particles are separated into groups, then there would be as 
many different games as the total number of possible group formations [33].)

The dynamic noncooperative game played by $\mathrm{N}$ agents is

$$
\min _{u^{i}}\left\{L^{i}\right\} \text { subject to } \dot{x}^{i}=u^{i} \quad \forall i=1, \ldots, N
$$

The problem that faces each agent is an optimal control problem and necessary conditions are obtained by Pontryagin's minimum principle (see [27, Theorem 6.11] and [34]). A Nash equilibrium solution exists provided the optimal solutions of $\mathrm{N}$ agents result, when simultaneously considered, in well defined position trajectories for given $x^{i}(0) \in \mathbf{R}, i=1, \ldots, N$ [27, Section VI-C]. Here, we limit the permissible strategies $u^{i}(t)=\dot{x}^{i}(t)$ available to agents to be continuous with respect to the initial conditions $x^{i}(0)$ (see [27, p. 227] and [35]). We will refer to a solution of this problem as a Nash equilibrium with free terminal state.

Note that in defining the above game, we have not specified the foraging target (food supply) location but added a simple quadratic term to the cost functional that penalizes the distances to the target location, which is the origin $x^{i}=0$ for $i=1, \ldots, N$. A solution, if it exists, should have the property that the swarm gets progressively closer to the origin. The specification of the origin as the target would mean that each agent knows the exact target location at the outset. Thus, if we consider the same cost functional (1), but without the first term, and specify $x^{i}(T)=0$ for $i=1, \ldots, N$ as the terminal condition, then we obtain a different game and a new problem. We will refer to a solution of this new problem as a Nash equilibrium with specified terminal condition.

\section{MAIN RESULTS}

A Nash equilibrium, if it exists, is shown in the Appendix to be a solution of a nonlinear differential equation (13) in terms of positions of the agents. Since this differential equation does not obey any local Lipschitz condition, the existence and uniqueness of a solution is not evident. Existence and uniqueness in dynamic games is a difficult problem, and as an example of a result on existence and uniqueness in a simpler problem than the one considered here, see [36].

The results below show that there is a unique Nash equilibrium with free or specified terminal condition for every initial position for the agents. These equilibria display many known characteristics of a swarm behavior. Explicit expressions for instantaneous pairwise distances between agents, the swarm size, and the distance of the swarm center to the foraging location are derived.

\section{A. Free Terminal Condition}

Theorem 1: A Nash equilibrium with free terminal condition of the game (2) and (1) exists, is unique, and is such that the initial ordering among the $N$ agents in the queue is preserved during $[0, T]$. The Nash solution has the following properties.
P1: The distance between any two agents $i, j$ at time $t$ is given by

$$
\begin{aligned}
x^{i}(t)-x^{j}(t)= & \frac{v_{a t t}(t, T)}{\Delta(T)}\left[x^{i}(0)-x^{j}(0)\right] \\
& +r \frac{v_{r e p}(t, T)}{\Delta(T)}\left[s^{i}(0)-s^{j}(0)\right]
\end{aligned}
$$

where with $\alpha:=\sqrt{N a}$

$$
\begin{aligned}
& \Delta(T):=\alpha \cosh (\alpha T)+f \sinh (\alpha T) \\
& v_{a t t}(t, T):=\alpha \cosh [\alpha(T-t)]+f \sinh [\alpha(T-t)] \\
& v_{r e p}(t, T):=\frac{1}{2 \alpha}\left[h_{1}(t, T)+h_{2}(t, T)\right] \\
& h_{1}(t, T):=f\{\sinh (\alpha T)-\sinh (\alpha t)-\sinh [\alpha(T-t)]\} \\
& h_{2}(t, T):=\alpha\{\cosh (\alpha T)-\cosh [\alpha(T-t)]\} \\
& s^{i}(0):=\sum_{k=1, k \neq i}^{N} \operatorname{sgn}\left[x^{i}(0)-x^{k}(0)\right], i=1, \ldots, N .
\end{aligned}
$$

P2: For every $T$ and as $T \rightarrow \infty$, the swarm size $d(t):=$ $\max _{i, j}\left|x^{i}(t)-x^{j}(t)\right|$ remains bounded in $[0, T]$

$$
\begin{aligned}
d(t)= & \frac{v_{\text {att }}(t, T)}{\Delta(T)} d(0)+\frac{v_{r e p}(t, T)}{\Delta(T)} r m(0) \\
& \leq \frac{v_{a t t}\left(t^{*}, T\right)}{\Delta(T)} d(0)+\frac{v_{r e p}\left(t^{*}, T\right)}{\Delta(T)} r m(0)
\end{aligned}
$$

where

$$
\begin{gathered}
t^{*}=\frac{1}{2 \alpha} \ln \\
\left(\frac{e^{\alpha T}\left\{\left[f\left(e^{\alpha T}-1\right)+\alpha e^{\alpha T}\right] r m(0)-2 \alpha^{2}(f+\alpha) e^{\alpha T} d(0)\right\}}{\left[f\left(e^{\alpha T}-1\right)+\alpha\right] r m(0)+2 \alpha^{2}(f-\alpha) d(0)}\right) .
\end{gathered}
$$

$d(0):=\max _{i, j}\left|x^{i}(0)-x^{j}(0)\right|$ is the distance between the first and the last agent in the queue at the initial time, and $m(0):=$ $\max _{i, j}\left|s^{i}(0)-s^{j}(0)\right|=2 N-2$. The bound is attained if and only if $0 \leq t^{*} \leq T$. Maximum swarm size is attained at 0 if $t^{*}<0$.

The expression for the swarm size at the final time is

$$
\begin{aligned}
& d(T)=\frac{\cosh (\alpha T)-1}{2[\alpha \cosh (\alpha T)+f \sinh (\alpha T)]} r m(0) \\
& +\frac{\alpha \cosh (\alpha T)+f \sinh (\alpha T)}{\alpha \cos (0) .}
\end{aligned}
$$

P3: The swarm center $\bar{x}(t):=\frac{x^{1}(t)+\ldots+x^{N}(t)}{N}$ is given by

$$
\bar{x}(t)=\bar{x}(0)\left(1-\frac{f t}{T f+1}\right)
$$

which monotonically approaches the origin as $t \rightarrow T$ and ends up at the origin as $T \rightarrow \infty$.

P4: As $T \rightarrow \infty$, the distances between the consecutive agents in the queue are the same and are equal to $\frac{r}{2(\alpha+f)}$.

Remark 1: The main result above is that a swarming behavior, an act of aggregation, does follow from noncooperative actions of the $N$ agents. The fact that the game has a unique Nash equilibrium is also significant. The initial ordering of the agents in the queue is preserved at all times in this Nash solution. This is, of course, a consequence of the attraction and repulsion terms in each agent's cost functional, the effect of which turns out to be similar to connecting the agents in the queue to each other by translational springs [37]. 
Remark 2: The swarm size throughout the foraging activity is given in (P2). The foraging activity of the swarm is accomplished increasingly better given sufficient time by (P3). In (P1), an explicit expression is given for pairwise distances. It is also possible to describe the individual paths $x^{i}(t)$ explicitly. However, the formula is rather lengthy and is not included here. By $(\mathrm{P} 4)$, given sufficient time, the foraging swarm will be more regular as it gets closer to the foraging location since distances between adjacent agents will be progressively more uniform. A closer examination of $d(T)$ reveals an additional property of the swarm. If the agents start far apart from each other at the initial time, then the attraction term becomes effective and they end up being closer together at the final time. Conversely, if they start close enough together, then the repulsion term is more effective and they later get apart from each other.

\section{B. Specified Terminal Condition}

The Nash equilibrium with specified terminal condition $x^{i}(T)=0$ for $i=1, \ldots, N$ is described next. We remark that the expressions for distances between agents, swarm size, swarm center, etc., are quite different from those in Theorem 1. This is because, due to the difference in the terminal condition, a new (but related) game is obtained.

Theorem 2: A Nash equilibrium with specified terminal condition exists, is unique, and is such that the initial ordering among the $N$ agents in the queue is preserved during [0,T]. The Nash solution has the following properties.

P1: The distance between any two agents $i, j$ at time $t$ is given by

$$
\begin{aligned}
x^{i}(t)-x^{j}(t)= & \frac{w_{\text {att }}(t, T)}{\Lambda(T)}\left[x^{i}(0)-x^{j}(0)\right] \\
& +r \frac{w_{r e p}(t, T)}{\Lambda(T)}\left[s^{i}(0)-s^{j}(0)\right]
\end{aligned}
$$

where

$$
\begin{aligned}
& \Lambda(T):=\sinh (\alpha T) \\
& w_{\text {att }}(t, T):=\sinh [\alpha(T-t)] \\
& w_{\text {rep }}(t, T):=\frac{1}{2 \alpha}\{\sinh (\alpha T)-\sinh (\alpha t)-\sinh [\alpha(T-t)]\} .
\end{aligned}
$$

$P 2$ : For every $T$ and as $T \rightarrow \infty$, the swarm size $d(t):=$ $\max _{i, j}\left|x^{i}(t)-x^{j}(t)\right|$ remains bounded in $[0, T]$

$$
\begin{aligned}
d(t)= & \frac{w_{\text {att }}(t, T)}{\Lambda(T)} d(0)+\frac{w_{\text {rep }}(t, T)}{\Lambda(T)} r m(0) \\
& \leq \frac{w_{\text {att }}\left(t^{*}, T\right)}{\Lambda(T)} d(0)+\frac{w_{\text {rep }}\left(t^{*}, T\right)}{\Lambda(T)} r m(0)
\end{aligned}
$$

where

$$
t^{*}=\frac{1}{2 \alpha} \ln \left(\frac{e^{\alpha T}\left[\left(e^{\alpha T}-1\right) r m(0)-2 \alpha^{2} e^{\alpha T} d(0)\right]}{\left(e^{\alpha T}-1\right) r m(0)+2 \alpha^{2} d(0)}\right) .
$$

The bound is attained if and only if $0 \leq t^{*} \leq T$. Maximum swarm size is attained at 0 if $t^{*}<0$. The swarm size at the final time is $d(T)=0$.

P3: The swarm center is given by

$$
\bar{x}(t)=\bar{x}(0)\left(1-\frac{t}{T}\right)
$$

so that $\bar{x}(T)=0$.
Remark 3: It will be noticed that the above expressions are all obtained by letting $f \rightarrow \infty$ in the corresponding expressions of Theorem 1. This is somewhat expected. Specifying the cost of each agent being away from the target location as infinity is as good as requiring that each agent is exactly at that location at the terminal time. The expressions of Theorem 2 are, nevertheless, derived independent of Theorem 1 in the Appendix by solving the game with the specified terminal condition.

Remark 4: Properties (P1)-(P3) of Theorem 2 show that the swarm that is formed with specified terminal condition has entirely similar features to the swarm formed with free terminal condition; the major difference is that the foraging target is reached exactly at the final time, as specified in the setup of the game.

\section{Dense Versus Sparse Swarms}

The degree of cohesion in our swarm model can be tuned by the levels of attraction and repulsion between the agents. The model is flexible in the sense that it can result in both dense and sparse swarms by selecting different values for the attraction constant $a$ and the repulsion constant $r$. It is expected that if the ratio $\frac{a}{r}$ increases, then the swarm will get denser, and the swarm will get sparser as it decreases, which is confirmed by the following result.

Corollary 1: a) The maximum swarm size is always attained in the interval $[0, T)$ and $b)$ the swarm size monotonically decreases in the interval $[0, T]$ if and only if

$$
\begin{gathered}
\frac{a}{r} d(0) \geq \frac{N-1}{N} \frac{\left(e^{\alpha T}-1\right)^{2} f+\alpha\left(e^{2 \alpha T}-1\right)}{\left(e^{2 \alpha T}+1\right) f+\alpha\left(e^{2 \alpha T}-1\right)} \\
\frac{a}{r} d(0) \geq \frac{N-1}{N} \frac{\left(e^{\alpha T}-1\right)^{2}}{e^{2 \alpha T}+1}
\end{gathered}
$$

in the free and specified terminal condition cases, respectively.

Thus, by a) and b), the value obtained when equality is achieved in (10) or in (11) is a critical value of the ratio $\frac{a}{r}$. The maximum swarm size is attained at $t=0$ for values larger than this critical value and it is attained in the open interval $(0, T)$ for values smaller. Note that this conclusion follows by the fact that the right-hand sides in both (10) and (11) are less than 1 for each value of $t \in[0, T]$ and for all values of $\alpha=\sqrt{N a}$. An asymptotic analysis of a) and b) indicates that swarm size, the pairwise distances (3), and (7) all grow hyperbolically and parabolically with time for $\frac{a}{r}$ sufficiently large and small, respectively.

\section{Simulations}

Simulations have been performed to verify the formulas derived in Theorems 1 and 2 and to detect other features of the swarming behavior than those already mentioned above.

The simulations were conducted for $N=10$ agents for random initial conditions between 0 and 1 . The swarm model parameters were selected as $f=1 / 2, a=1 / 2$, and $r=1$. The simulation duration was chosen as $T=1$. The same set of initial conditions and parameters were used for the two cases to make comparisons unbiased. Examples of the optimal 


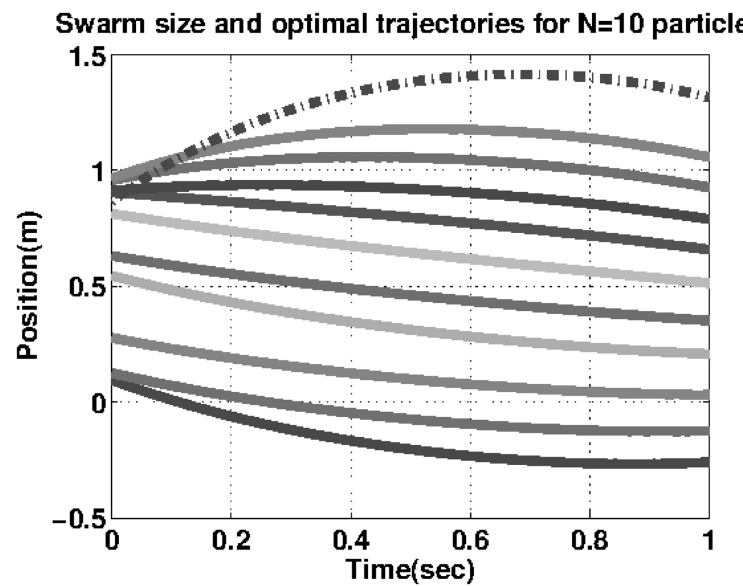

Fig. 1. Optimal trajectories and the swarm size for ten agents for the free terminal condition case.

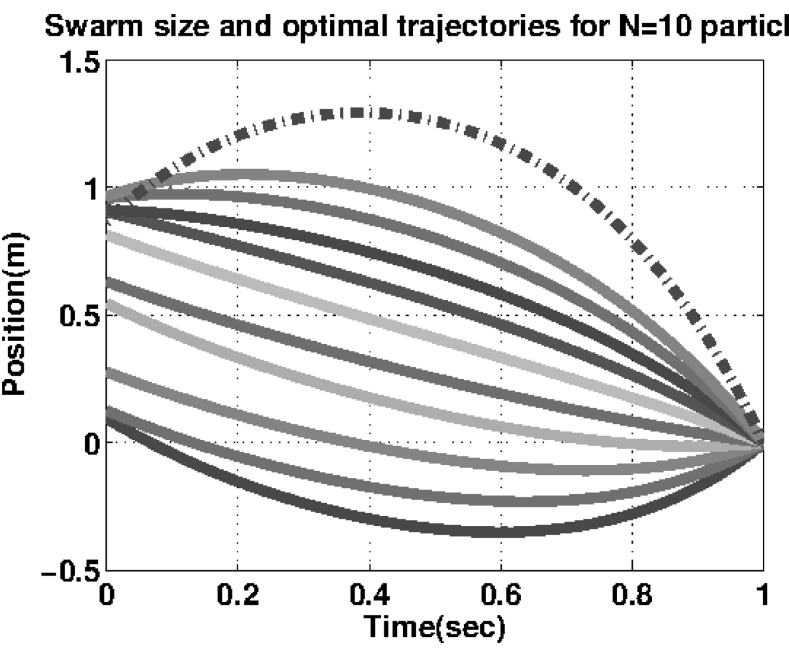

Fig. 2. Optimal trajectories and the swarm size for ten agents for the specified terminal case.

trajectories are shown in Figs. 1 and 2 for one set of initial conditions. The swarm size plots are shown with dashed lines in these figures. The features observed were similar in all other simulations.

The maximum swarm size for the free terminal state case is 1.4149 and the corresponding value for the specified terminal state case is 1.2938. All the predicted features by Theorems 1 and 2 are confirmed. One additional observation in the free terminal case is that no matter how nonuniform the swarm initial conditions are, the swarm evolves into a regular form, i.e., the distances between consecutive agents get more uniform after some time [38]. It is an important property of formation control [39] that the regularity is also maintained, given sufficient time in the case of departures or new entries into the swarm. This is also verified in Fig. 3 in which three individuals depart at second 1 from the agents of Fig. 1 and two new agents join the swarm at second 2 .

Although formal expressions for the inputs $u^{i}(t)=\dot{x}^{i}(t)$ 's can be derived, these are lengthy and not given here. However, in all simulations, their sizes remain within reasonable limits. For instance, in the free terminal state simulation above, the plots of inputs (velocities of the agents) are as given in Fig. 4.

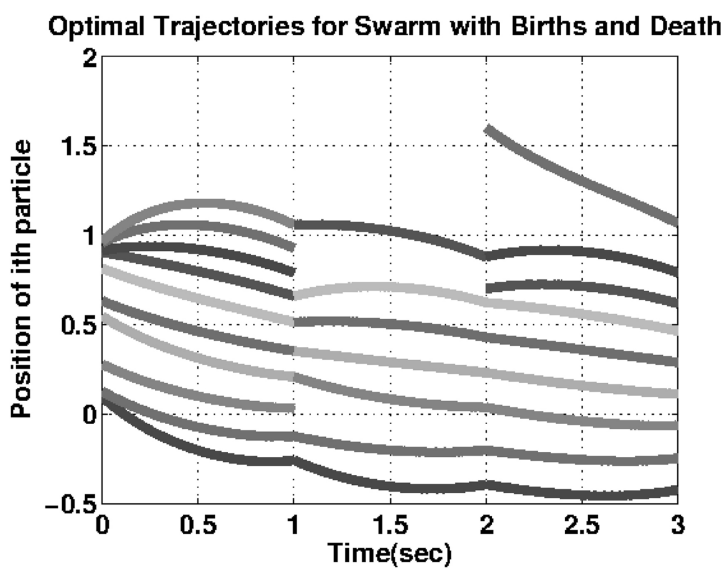

Fig. 3. Optimal trajectories for a swarm with departures and arrivals at different times.

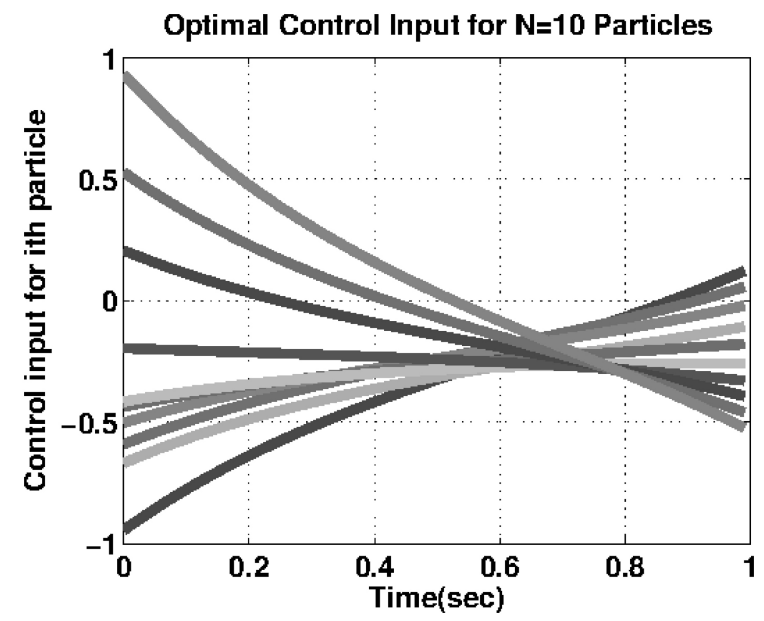

Fig. 4. Optimal control inputs (velocities) for the free terminal condition case.

Note that the sign of the velocities changes for each agent and the change occurs at a different time instant for each agent. This implies that each agent changes direction during foraging activity. This can also be seen in Figs. 1 and 2 as the positions first increase and then decrease after reaching a maximum at $t^{*}$ in the open interval $(0, T)$. The change of direction during swarming is a well known phenomenon commonly observed in many actual swarms [40]. For instance, abrupt changes occur in the direction of birds in the foraging flocks. After their maneuvers, the agents start navigating toward the foraging location. In Fig. 4, an example of such a behavior is observed, but in the case of a 1-D motion.

Simulations also indicate that the Nash equilibria are still confined to those in which initial ordering is preserved even if the strategy spaces of agents are enlarged to include those that are discontinuous with respect to initial positions. A theoretical justification of this observation is left for future work.

\section{CONCLUSION}

The dynamic game model introduced in this paper can be generalized in different directions. The first interesting generalization would be to extend the results on the 1-D 
motion presented here to 2-D and 3-D spaces. The model would then be applicable to robot motion planning. The second generalization would be to relax the assumption that every agent knows the location of every other agent and to examine whether the game in which every agent only knows the location of adjacent agents in the queue has a Nash solution. Furthermore, the cost functionals used by the agents and the foraging terms in them can be made more general to cover other interesting objectives for each agent. The main result of this study, that a swarming behavior can result as a Nash equilibrium of a noncooperative game played by individuals, is expected to be true in all these generalizations.

We have assumed in our game that the agents are identical, having the same attraction, repulsion, and foraging parameters - a reasonable assumption for biological swarms. However, the behavior obtained for nonidentical agents would still be of interest since it may clarify how essential the likeness of agents is for obtaining a swarming behavior.

\section{APPENDIX}

\section{HAMILTON-JACOBI FORMULATION}

The optimal control problem that faces the $i$ th agent is first considered. Introducing the Lagrange multiplier $p^{i}(t)$ and minimizing the Hamiltonian

$$
H^{i}=\sum_{j=1, j \neq i}^{N}\left(a\left(x^{i}-x^{j}\right)^{2}-r\left|x^{i}-x^{j}\right|\right)+\left(u^{i}\right)^{2}+p^{i} u^{i}
$$

leads to the necessary conditions

$$
\begin{aligned}
& u^{i}=\frac{-p^{i}}{2} \\
& \dot{p}^{i}=2 a(1-N) x^{i}+\sum_{j=1, j \neq i}^{N}\left(2 a x^{j}+\frac{r\left(x^{i}-x^{j}\right)}{\left|x^{i}-x^{j}\right|}\right) \\
& \dot{x}^{i}=u^{i}
\end{aligned}
$$

and the boundary conditions

$$
x^{i}(0) \in R, \quad p^{i}(T)=2 f x^{i}(T)
$$

for free and $x^{i}(T)=0$ for the specified terminal condition case.

Let $\mathcal{I}$ denote the matrix with all entries equal to 1 . Equation (12) for all $i=1, \ldots, N$ combined can be written as

$$
\left[\begin{array}{c}
\dot{\mathbf{x}} \\
\dot{\mathbf{p}}
\end{array}\right]=\left[\begin{array}{cc}
0 & \frac{-I}{2} \\
2 a(\mathcal{I}-N I) & 0
\end{array}\right]\left[\begin{array}{l}
\mathbf{x}(t) \\
\mathbf{p}(t)
\end{array}\right]+r\left[\begin{array}{c}
0 \\
\mathbf{s}(t)
\end{array}\right]
$$

where

$$
\begin{aligned}
& \mathbf{x}:=\left[\begin{array}{lll}
x^{1} & \ldots & x^{N}
\end{array}\right]^{T} \quad \mathbf{p}:=\left[\begin{array}{lll}
p^{1} & \ldots & p^{N}
\end{array}\right]^{T} \\
& \mathbf{s}:=\left[\sum_{j=1, j \neq 1}^{N} \operatorname{sgn}\left(x^{1}-x^{j}\right) \quad \ldots \quad \sum_{j=1, j \neq N}^{N} \operatorname{sgn}\left(x^{N}-x^{j}\right)\right]^{T} .
\end{aligned}
$$

The signum vector $\mathbf{s}$ is piecewise-constant in the interval $[0, T]$ with each constant value obtained by a permutation of entries in $\left[\begin{array}{llllll}1-N & 3-N & \ldots & N-3 & N-1\end{array}\right]^{T}$. This is because its $i$ th entry $s^{i}=\sum_{j=1, j \neq i}^{N} \operatorname{sgn}\left(x^{i}-x^{j}\right)$ is equal to $2 B(i)+1-N$, where $B(i)$ denotes the number of agents behind the agent $i$ and can assume a value between 0 and $N-1$. Note that the vector $\mathbf{s}$ in (13) originates from the repulsion terms in the cost functionals so that the part of the solution obtained with $\mathbf{s}=0$ will be called the attraction term and summand due to $\mathbf{s}$, the repulsion term. Thus

$$
\left[\begin{array}{l}
\mathbf{x}(t) \\
\mathbf{p}(t)
\end{array}\right]=\left[\begin{array}{l}
\mathbf{x}_{\text {att }}(t) \\
\mathbf{p}_{\text {att }}(t)
\end{array}\right]+\left[\begin{array}{l}
\mathbf{x}_{\text {rep }}(t) \\
\mathbf{p}_{\text {rep }}(t)
\end{array}\right]
$$

where

$$
\begin{aligned}
& {\left[\begin{array}{l}
\mathbf{x}_{\text {att }}(t) \\
\mathbf{p}_{\text {att }}(t)
\end{array}\right]=\left[\begin{array}{ll}
\phi_{11}(t) & \phi_{12}(t) \\
\phi_{21}(t) & \phi_{22}(t)
\end{array}\right]\left[\begin{array}{l}
\mathbf{x}(0) \\
\mathbf{p}(0)
\end{array}\right]} \\
& {\left[\begin{array}{l}
\mathbf{x}_{\text {rep }}(t) \\
\mathbf{p}_{\text {rep }}(t)
\end{array}\right]=\int_{0}^{t} r\left[\begin{array}{l}
\phi_{12}(t-\tau) \\
\phi_{22}(t-\tau)
\end{array}\right] \mathbf{s}(\tau) d \tau .}
\end{aligned}
$$

Here, the partitioned matrix $\phi(t)$ is the state transition matrix of (13) when $\mathbf{s}=0$. Its partitions can be computed to be given by

$$
\phi_{i j}(t)=a_{i j}(t) I+b_{i j}(t)(\mathcal{I}-I) \quad i, j=1,2
$$

where

$$
\begin{aligned}
& a_{11}(t)=a_{22}(t)=\frac{1}{N}+\frac{N-1}{N} \cosh (\alpha t) \\
& b_{11}(t)=b_{22}(t)=\frac{1}{N}[1-\cosh (\alpha t)] \\
& a_{12}(t)=-\frac{1}{2 N \alpha}[t \alpha+(N-1) \sinh (\alpha t)] \\
& b_{12}(t)=-\frac{1}{2 N \alpha}[\alpha t-\sinh (\alpha t)] \\
& a_{21}(t)=-\frac{2 \alpha(N-1)}{N} \sinh (\alpha t) \\
& b_{21}(t)=\frac{2 \alpha}{N} \sinh (\alpha t) .
\end{aligned}
$$

A solution for an arbitrary, but fixed, $\mathbf{x}(0) \in \mathbf{R}^{N}$ of the nonlinear differential equation (13) obeying the final condition $2 f \mathbf{x}(T)=\mathbf{p}(T)$, respectively, $\mathbf{x}(T)=\mathbf{0}$, is a Nash equilibrium with free, respectively, specified, terminal condition of the dynamic game (2).

Observe that each $\phi_{i j}(t)$ is a matrix with identical diagonal and identical off-diagonal entries. The sum, multiple, and inverse of such matrices inherit the same property as summarized in the following lemma, the proof of which is straightforward and is omitted.

Lemma A.1. Let $F:=\mathcal{I}-I \in \mathbf{R}^{N \times N}, \mathbf{v} \in \mathbf{R}^{1 \times N}$ be a row vector of all 1's, $\mathbf{w} \in \mathbf{R}^{1 \times N}$ be a row vector of all zeros except 1 and -1 in entries $i<j$, respectively. Also, let $a, b, c, d \in \mathbf{R}$. Then

$$
\begin{aligned}
& F^{2}=(N-1) I+(N-2) F \\
& (a I+b F)(c I+d F)=[a c+b d(N-1)] I \\
& \quad+[a d+b c+b d(N-2)] F \\
& (a I+b F)^{-1}=\frac{b F-[a+b(N-2)] I}{(b-a)[a+b(N-1)]} \\
& \operatorname{det}\{a I+b F\}=(a-b)^{N-1}[a+b(N-1)] \\
& \mathbf{v}(a I+b F)=[a+b(N-1)] \mathbf{v} \\
& \mathbf{w}(a I+b F)=(a-b) \mathbf{w} .
\end{aligned}
$$

Proofs of Theorems 1 and 2: We first establish the existence of a Nash equilibrium. Suppose that the initial ordering among 
agents is preserved so that $\mathbf{s}(t)=\mathbf{s}(0)$ for all $t \in[0, T]$. Then, the repulsion term in (15) can be written as

$$
\begin{aligned}
{\left[\begin{array}{l}
\mathbf{x}_{\text {rep }}(t) \\
\mathbf{p}_{\text {rep }}(t)
\end{array}\right]=} & \left(\int_{0}^{t} r\left[\begin{array}{l}
\phi_{12}(t-\tau) \\
\phi_{22}(t-\tau)
\end{array}\right] d \tau\right) \mathbf{s}(0) \\
=: & {\left[\begin{array}{l}
\psi_{1}(t, 0) \\
\psi_{2}(t, 0)
\end{array}\right] \mathbf{s}(0) }
\end{aligned}
$$

where

$$
\begin{aligned}
\psi_{i}(t, 0) & =p_{i}(t) I+q_{i}(t)(\mathcal{I}-I) \quad i=1,2 \\
p_{1}(t) & =-\frac{t^{2}}{4 N}-\frac{N-1}{N \alpha^{2}} \sinh ^{2}\left(\frac{\alpha t}{2}\right) \\
q_{1}(t) & =-\frac{t^{2}}{4 N}+\frac{1}{N \alpha^{2}} \sinh ^{2}\left(\frac{\alpha t}{2}\right) \\
p_{2}(t) & =\frac{t}{N}+\frac{N-1}{N \alpha} \sinh (\alpha t) \\
q_{2}(t) & =\frac{1}{N \alpha}[\alpha t-\sinh (\alpha t)] .
\end{aligned}
$$

Derivation of $(P 1)-(P 4)$ of Theorem 1: Using the boundary condition $\mathbf{p}(T)=2 f \mathbf{x}(T)$ in (14) for $t=T$ gives

$$
\begin{aligned}
& {\left[2 f \phi_{11}(T)-\phi_{21}(T)\right] \mathbf{x}(0)+\left[2 f \phi_{12}(T)-\phi_{22}(T)\right] \mathbf{p}(0)} \\
& +\left[2 f \psi_{1}(T, 0)-\psi_{2}(T, 0)\right] \mathbf{s}(0)=0
\end{aligned}
$$

which can be solved for $\mathbf{p}(0)$ if $2 f \phi_{12}(T)-\phi_{22}(T)$ is nonsingular. By Lemma A.1, the determinant of $2 f \phi_{12}(T)-\phi_{22}(T)$ is obtained to be $\frac{\alpha(f t+1)\left(-\cosh (\alpha t)-\frac{f \sinh (\alpha t)}{\alpha}\right)^{N}}{f \sinh (\alpha t)+\alpha \cosh (\alpha t)}$, which is easily seen to be nonzero for all $\alpha>0, f>0$. Thus, $2 f \phi_{12}(T)-\phi_{22}(T)$ is invertible and there is a candidate solution of (13) for every $\mathbf{x}(0)$. This solution is

$$
\begin{aligned}
& \mathbf{x}(t)= \\
& \left\{\phi_{11}(t)-\phi_{12}(t)\left[2 f \phi_{12}(T)-\phi_{22}(T)\right]^{-1}\left[2 f \phi_{11}(T)-\phi_{21}(T)\right]\right\} \\
& \mathbf{x}(0)+\left\{\psi_{1}(t, 0)-\phi_{12}(t)\left[2 f \phi_{12}(T)-\phi_{22}(T)\right]^{-1}\left[2 f \psi_{1}(T, 0)\right.\right. \\
& \left.\left.-\psi_{2}(T, 0)\right]\right\} \mathbf{s}(0) .
\end{aligned}
$$

In this expression, by Lemma A.1, the coefficient matrices of $\mathbf{x}(0)$ and $\mathbf{s}(0)$ have identical diagonal/off-diagonal entries. Multiplying each term in (19) by the row vector $\mathbf{w}$ of Lemma A.1 and employing the left eigenvector property of $\mathbf{w}$ given there, the simple expressions (3) for pairwise distances are obtained.

A crucial step is to verify that for any pair $i, j$

$$
\operatorname{sgn}\left[x^{i}(t)-x^{j}(t)\right]=\operatorname{sgn}\left[x^{i}(0)-x^{j}(0)\right]
$$

for all $t \in[0, T]$. To see this, we first note that $s^{i}(0)-s^{j}(0)=$ $2 r[B(i)-B(j)]$ so that $\operatorname{sgn}\left[s^{i}(0)-s^{j}(0)\right]=\operatorname{sgn}\left[x^{i}(0)-x^{j}(0)\right]$. Next, we note that $v_{a t t}(t)>0$ and $\Delta(T)>0$ since they are linear combinations of hyperbolic functions which are positive for positive arguments. Also, $v_{\text {rep }}(t)>0$ for all $t \in(0, T]$, since $v_{\text {rep }}(0)=0$ and $v_{\text {rep }}(T)>0$ and the coefficient of the highest degree term in the quadratic parabola of $v_{\text {rep }}(t)$ is negative which indicates that $v_{\text {rep }}(t)$ is a parabola oriented to $-\infty$ that is positive between 0 and $T$. This proves that (19) is indeed a solution.
The expression in (P2) and (P3) of Theorem 1 will now be derived. The swarm size at any $t \in[0, T]$ is given by

$$
\begin{aligned}
d(t) & =\frac{v_{\text {att }}(t, T)}{\Delta(T)} \max _{i, j}\left[x^{i}(0)-x^{j}(0)\right] \\
& +\frac{v_{\text {rep }}(t, T)}{\Delta(T)} r \max _{i, j}\left[s^{i}(0)-s^{j}(0)\right] \\
& =\frac{v_{\text {att }}(t, T)}{\Delta(T)} d(0)+\frac{v_{\text {rep }}(t, T)}{\Delta(T)} r \max _{i, j}\left[s^{i}(0)-s^{j}(0)\right]
\end{aligned}
$$

where $\max _{i, j}\left[s^{i}(0)-s^{j}(0)\right]$ is the difference between the first and last agent's signum numbers, respectively, $N-1$ and $1-N$. This yields $m(0)=\max _{i, j}\left[s^{i}(0)-s^{j}(0)\right]=2 N-2$ and

$$
d(t)=\frac{v_{\text {att }}(t, T)}{\Delta(T)} d(0)+\frac{v_{r e p}(t, T)}{\Delta(T)} r(2 N-2) .
$$

Maximizing this expression, it is easily shown that maximum is attained at $t^{*}$ of (P2) if $t^{*} \in(0, T)$, at $t=0$ if $t^{*} \leq 0$, and at $t=T$ if $t^{*} \geq T$. The expression in (P2) at the final time is obtained by evaluating (21) at $t=T$.

The expression for the swarm center in (P3) is obtained from (19) by multiplying each term on the left by $N^{-1} \mathbf{v}$, where $\mathbf{v}$ is the row vector of Lemma A.1, the average of the entries of $\mathbf{x}(t)$ is obtained and yields (6).

The last property (P4) follows by (3), where $i$ and $j$ are taken as consecutive agents in the queue, evaluating at $t=T$ and by taking the limit as $T \rightarrow \infty$. Note that if $i, j$ are two consecutive agents with $j$ behind $i$, then $s^{i}(0)-s^{j}(0)=1$. This gives

$$
x^{i}(T)-x^{j}(T)=\frac{\alpha}{\Delta(T)}\left[x^{i}(0)-x^{j}(0)\right]+r \frac{\cosh (\alpha T)-1}{2 \Delta(T)}
$$

so that in the limit $T \rightarrow \infty$, the distance between agents $i$ and $j$ is indeed $\frac{r}{2(\alpha+f)}$.

Derivation of $(P 1)-(P 3)$ of Theorem 2: Using the boundary condition $\mathbf{x}(T)=0$ in (14) for $t=T$ gives

$$
\phi_{11}(T) \mathbf{x}(0)+\phi_{12}(T) \mathbf{p}(0)+\psi_{1}(T, 0) \mathbf{s}(0)=0
$$

which can be solved for $\mathbf{p}(0)$ since $\phi_{12}(T)$ is nonsingular. It follows that there is a candidate solution of (13) for every $\mathbf{x}(0)$. This solution is

$$
\begin{aligned}
\mathbf{x}(t)= & \left\{\phi_{11}(t)-\phi_{12}(t)\left[\phi_{12}(T)\right]^{-1} \phi_{11}(T)\right\} \mathbf{x}(0) \\
& +\left\{\psi_{1}(t, 0)-\phi_{12}(t)\left[\phi_{12}(T)\right]^{-1} \psi_{1}(T, 0)\right\} \mathbf{s}(0) .
\end{aligned}
$$

Again, the coefficient matrices of $\mathbf{x}(0)$ and $\mathbf{s}(0)$ have identical diagonal/off-diagonal entries by Lemma A.1 so that proceeding similarly to the case of Theorem 1, expressions (7) for pairwise distances are obtained from (22).

In order to verify (20), we again observe that $\operatorname{sgn}\left[s^{i}(0)-\right.$ $\left.s^{j}(0)\right]=\operatorname{sgn}\left[x^{i}(0)-x^{j}(0)\right]$ and note that $w_{\text {att }}(t)>0, \Delta(T)>0$ since both are hyperbolic functions that are positive for positive arguments. Also, $w_{\text {rep }}(t)>0$ for all $t \in(0, T]$, since $w_{\text {rep }}(0)=0$ and $w_{\text {rep }}(T)=0$ and the coefficient of the highest degree term in the quadratic parabola of $v_{\text {rep }}(t)$ is negative which indicates that $w_{\text {rep }}(t)$ is a parabola oriented to $-\infty$ that is positive between 0 and $T$. This proves that (19) is indeed a solution.

The expressions in (P2) and (P3) of Theorem 2 are obtained by a similar procedure to that in the proof of Theorem 2 . 
Uniqueness: We now prove that the solutions given in Theorems 1 and 2 are actually unique solutions of the two games (2) with respect to strategies that are continuous against initial positions. We only prove the uniqueness of Nash equilibrium for the free terminal condition case as the proof in the case of specified terminal condition is similarly done.

If the ordering of the agents in the queue is not the same during the interval $[0, T]$, then there is a time $t_{k} \in(0, T)$ at which $x^{i}\left(t_{k}\right)=x^{j}\left(t_{k}\right)$ for some $i \neq j$. Because the terminal conditions at time $T$ are to be satisfied exactly (not asymptotically), the number of changes in the ordering in the queue must be finite in number, i.e., there is a finite $n$ and $t_{1}, \ldots, t_{n-1} \in(0, T)$ such that $x^{i}\left(t_{k}\right)=x^{j}\left(t_{k}\right)$ for some $i \neq j$ for $k=1, \ldots, n-1$ and the ordering is unchanged in each interval $\left(t_{k-1}, t_{k}\right) k=1, \ldots, n$. Let $t_{0}:=0, t_{n}:=T$. It follows that the signum vector $\mathbf{s}(t)$ has constant value $\mathbf{s}_{k-1} \in \mathbf{R}^{N}$ in each interval $\left(t_{k-1}, t_{k}\right)$ for $k=1, \ldots, n$. Here, we assume that there is also a $k \in\{0,1, \ldots, n\}$ and $i \neq j$ at which $x^{i}\left(t_{k}\right) \neq x^{j}\left(t_{k}\right)$, since the singular case $x^{i}\left(t_{k}\right)=x^{j}\left(t_{k}\right)$ for all $i, j, k$ is covered by the Nash equilibrium in which $\mathbf{s}_{k}=\mathbf{0}$ for all $k \in\{0,1, \ldots, n\}$. (Agents start and travel glued together in the whole interval $[0, T]$.) Then, for $t \in\left(t_{k-1}, t_{k}\right)$, we have

$$
\begin{aligned}
\int_{t_{k-1}}^{t} & {\left[\begin{array}{l}
\phi_{12}(t-\tau) \\
\phi_{22}(t-\tau)
\end{array}\right] r \mathbf{s}(\tau) d \tau=\left(\int_{t_{k-1}}^{t} r\left[\begin{array}{l}
\phi_{12}(t-\tau) \\
\phi_{22}(t-\tau)
\end{array}\right] d \tau\right) \mathbf{s}_{k-1} } \\
= & {\left[\begin{array}{l}
\psi_{1}\left(t_{k-1}, t\right) \\
\psi_{2}\left(t_{k-1}, t\right)
\end{array}\right] \mathbf{s}_{k-1}=\psi\left(t_{k-1}, t\right) \mathbf{s}_{k-1} }
\end{aligned}
$$

so that the solution of (15) is

$$
\mathbf{z}(t)=\phi\left(t-t_{k-1}\right) \mathbf{z}\left(t_{k-1}\right)+\psi\left(t, t_{k-1}\right) \mathbf{s}_{k-1} \quad t \in\left[t_{k-1}, t_{k}\right)
$$

for $k=1, \ldots, n$. The solution in terms of the initial value $\mathbf{z}(0)$ is

$$
\begin{aligned}
\mathbf{z}(t) & =\phi(t) \mathbf{z}(0)+\psi\left(t, t_{k-1}\right) \mathbf{s}_{k-1} \\
& +\sum_{l=1}^{k-1} \phi\left(t-t_{l}\right) \psi\left(t_{l}, t_{l-1}\right) \mathbf{s}_{l-1} \quad t \in\left[t_{k-1}, t_{k}\right)
\end{aligned}
$$

for $k=1, \ldots, n$. Employing the terminal condition $\mathbf{p}(T)=$ $2 f \mathbf{x}(T)$, multiplying each term in (23) (with $t=T$ ) on the left by $\left[\begin{array}{ll}2 f I & -I\end{array}\right]$, and using the fact that $2 f \phi_{12}(T)-\phi_{22}(T)$ is invertible, we determine

$$
\begin{aligned}
& \mathbf{p}(0)=-\left[2 f \phi_{12}(T)-\phi_{22}(T)\right]^{-1}\left\{\left[2 f \phi_{11}(T)-\phi_{21}(T)\right] \mathbf{x}(0)\right. \\
& \left.+\sum_{l=1}^{n}\left[\begin{array}{ll}
2 f I & -I
\end{array}\right] \phi\left(T-t_{l}\right) \psi\left(t_{l}, t_{l-1}\right) \mathbf{s}_{l-1}\right\} .
\end{aligned}
$$

Substituting in (23) and solving for $\mathbf{x}(t)$ for $t \in\left[0, t_{1}\right)$, we get

$$
\begin{aligned}
& \mathbf{x}(t)= \\
& \left\{\phi_{11}(t)-\phi_{12}(t)\left[2 f \phi_{12}(T)-\phi_{22}(T)\right]^{-1}\left[2 f \phi_{11}(T)-\phi_{21}(T)\right]\right\} \\
& \mathbf{x}(0)+\sum_{l=1}^{n} \Gamma_{l}\left(t, t_{0}, \ldots, t_{n}\right) \mathbf{s}_{l-1}
\end{aligned}
$$

for some functions $\Gamma_{l}\left(t, t_{0}, \ldots, t_{n}\right)$ for $l=1, \ldots, n$ [independent of $\mathbf{x}(0)]$. At $t=t_{1}$, the first time instant at which the ordering changes, we have $x^{i}\left(t_{k}\right)=x^{j}\left(t_{k}\right)$ for some $i<j$. Multiplying each term in (24) on the left by the row vector of all zeros except 1 at $i$ th and -1 at the $j$ th entry, we get

$$
\begin{aligned}
x^{i}(t)-x^{j}(t) & -\frac{v_{a t t}(t, T)}{\Delta(T)}\left[x^{i}(0)-x^{j}(0)\right] \\
& =\sum_{l=1}^{n} \frac{v_{r e p}^{l}\left(t, t_{0}, \ldots, t_{n}\right)}{\Delta(T)} \mathbf{s}_{l-1}
\end{aligned}
$$

for some functions $v_{r e p}^{l}\left(t, t_{0}, \ldots, t_{n}\right)$ that are independent of $\mathbf{x}(0)$. In this equality, the first term can be made as small as desired by choosing $t=t_{1}+\epsilon$ for $\epsilon$ sufficiently small. As $\mathbf{x}(0)$ is perturbed, the last term can assume only a finite number of values since $\mathbf{s}_{l}$ has only a finite number of values for $l=1, \ldots, n-1$. Since, by continuity of strategies, the lefthand side assumes an infinity of values for perturbed values of $\mathbf{x}(0)$, it follows that the equality cannot hold. This implies that a solution that has a change of ordering in $(0, T)$ cannot be a solution of (13). Therefore, there is a unique Nash equilibrium.

Proof of Corollary 1:

a) From the proof of Theorem 1, we know that the maximum swarm size is at $t=T$ if and only if $t^{*} \geq T$, which is the case if and only if

$$
\frac{\left[f\left(e^{\alpha T}-1\right)+\alpha e^{\alpha T}\right] r m(0)-2 \alpha^{2}(f+\alpha) e^{\alpha T} d(0)}{\left[f\left(e^{\alpha T}-1\right)+\alpha\right] r m(0)+2 \alpha^{2}(f-\alpha) d(0)} \geq e^{\alpha T}
$$

and

$$
\frac{e^{\alpha T}\left[\left(e^{\alpha T}-1\right) r m(0)-2 \alpha^{2} e^{\alpha T} d(0)\right]}{\left(e^{\alpha T}-1\right) r m(0)+2 \alpha^{2} d(0)} \geq e^{\alpha T}
$$

in free and specified terminal cases, respectively. Noting that the denominators are positive for all $t$ in both expressions, the inequality does not change direction when (25) or (26) is multiplied by its denominator. This gives that (25) and (26) are both equivalent to

$$
\left(e^{\alpha T}-1\right)^{2} r m(0)+4 \alpha^{2} e^{\alpha T} d(0) \leq 0
$$

which is only possible when $m(0)=0$ and $d(0)=0$, the singular solution of the swarm traveling glued together in the whole interval $[0, T]$.

b) Again, from the proof of Theorem 1, we know that the maximum swarm size is at $t=0$ if and only if $t^{*} \leq 0$, which is the case if and only if the left-hand sides of (25) or (26) are less than or equal to $e^{-\alpha T}$. Again, multiplying by their denominators, substituting $m(0)=2 N-2$, and organizing, these inequalities turn out to be equivalent to (10) and (11).

\section{REFERENCES}

[1] J. K. Parrish and L. Edelstein-Keshet, "Complexity, pattern, and evolutionary trade-offs in animal aggregation," Science, vol. 284, no. 5411, pp. 99-101, 1999.

[2] T. Vicsek and A. Zafeiris, "Collective motion," Phys. Rep., vol. 517, no. 3, pp. 71-140, 2012.

[3] D. Gu, "A differential game approach to formation control," IEEE Trans. Control Syst. Technol., vol. 16, no. 1, pp. 85-93, Jan. 2008.

[4] H. Weimerskirch, J. Martin, Y. Clerquin, P. Alexandre, and S. Jiraskova, "Energy saving in flight formation," Nature, vol. 413, no. 6857, pp. 697-698, 2001. 
[5] S.-J. Chung and J.-J. Slotine, "Cooperative robot control and concurrent synchronization of Lagrangian systems," IEEE Trans. Robot., vol. 25, no. 3, pp. 686-700, Jun. 2009.

[6] J. Mei, W. Ren, and G. Ma, "Distributed coordinated tracking with a dynamic leader for multiple Euler-Lagrange systems," IEEE Trans. Automatic Control, vol. 56, no. 6, pp. 1415-1421, Jun. 2011.

[7] Z. Meng, Z. Lin, and W. Ren, "Leader-follower swarm tracking for networked Lagrange systems," Syst. Control Lett., vol. 61, no. 1, pp. 117-126, 2012.

[8] L. Makarem and D. Gillet, "Decentralized coordination of autonomous vehicles at intersections," in Proc. World Congr., vol. 18, no. 1. 2011 pp. 13046-13051.

[9] S. LaValle and S. Hutchinson, "Optimal motion planning for multiple robots having independent goals," IEEE Trans. Robot. Autom., vol. 14, no. 6, pp. 912-925, Dec. 1998.

[10] H. Roozbehani, S. Rudaz, and D. Gillet, "On decentralized navigation schemes for coordination of multi-agent dynamical systems," in Proc. IEEE SMC, 2009, pp. 4807-4812.

[11] H. Roozbehani, S. Rudaz, and D. Gillet, "A Hamilton-Jacobi formulation for cooperative control of multi-agent systems," in Proc. IEEE SMC, 2009, pp. 4813-4818.

[12] C. Tomlin, G. Pappas, and S. Sastry, "Conflict resolution for air traffic management: A study in multiagent hybrid systems," IEEE Trans. Automatic Control, vol. 43, no. 4, pp. 509-521, Apr. 1998.

[13] C. Tomlin, G. Pappas, and S. Sastry, "Noncooperative conflict resolution [air traffic management]," in Proc. 36th IEEE Conf. Decision Control, vol. 2. 1997, pp. 1816-1821.

[14] C. Valicka, D. Stipanovic, S. Bieniawski, and J. Vian, "Cooperative avoidance control for UAVs," in Proc. 10th ICARCV, 2008, pp. $1462-1468$.

[15] C. Li, S. Yang, and T. T. Nguyen, "A self-learning particle swarm optimizer for global optimization problems," IEEE Trans. Syst., Man, Cybern. B, Cybern., vol. 42, no. 3, pp. 627-646, Jun. 2012.

[16] R. Xu, J. Xu, and D. Wunsch, "A comparison study of validity indices on swarm-intelligence-based clustering," IEEE Trans. Syst., Man, Cybern. B, Cybern., vol. 42, no. 4, pp. 1243-1256, Aug. 2012.

[17] V. Gazi, "Swarm aggregations using artificial potentials and slidingmode control," IEEE Trans. Robot., vol. 21, no. 6, pp. 1208-1214, Dec. 2005.

[18] W. Li, "Stability analysis of swarms with general topology," IEEE Trans. Syst., Man, Cybern. B, Cybern., vol. 38, no. 4, pp. 1084-1097, Aug. 2008.

[19] N. Leonard and E. Fiorelli, "Virtual leaders, artificial potentials and coordinated control of groups," in Proc. 40th IEEE Conf. Decision Control, vol. 3. 2001, pp. 2968-2973.

[20] P. Ogren, E. Fiorelli, and N. Leonard, "Cooperative control of mobile sensor networks: Adaptive gradient climbing in a distributed environment," IEEE Trans. Automatic Control, vol. 49, no. 8, pp. 1292-1302, Aug. 2004.

[21] L. A. Dugatkin and H. K. Reeve, Game Theory and Animal Behavior. Oxford, U.K.: Oxford Univ. Press, 1998.

[22] V. Gazi and K. M. Passino, "Stability analysis of social foraging swarms," IEEE Trans. Syst., Man, Cybern., vol. 34, no. 1, pp. 539-557, Feb. 2004.

[23] D. Chang, S. Shadden, J. Marsden, and R. Olfati-Saber, "Collision avoidance for multiple agent systems," in Proc. 42nd IEEE Conf. Decision Control, vol. 1. 2003, pp. 539-543.

[24] V. Gazi and K. Passino, "Stability analysis of swarms," IEEE Trans. Automatic Control, vol. 48, no. 4, pp. 692-697, Apr. 2003.

[25] S. Das, U. Halder, and D. Maity, "Chaotic dynamics in social foraging swarms: An analysis," IEEE Trans. Syst., Man, Cybern. B, Cybern., vol. 42, no. 4, pp. 1288-1293, Aug. 2012.

[26] J. Engwerda, LQ Dynamic Optimization and Differential Games. New York, NY, USA: Wiley, 2005

[27] T. Basar and G. J. Olsder, Dynamic Noncooperative Game Theory. Philadelphia, PA, USA: SIAM, 1999.

[28] L. Giraldeau and T. Caraco, Social Foraging Theory. Englewood Cliffs, NJ, USA: Princeton Univ. Press, 2000.

[29] C. Tomlin, J. Lygeros, and S. Shankar Sastry, "A game theoretic approach to controller design for hybrid systems," Proc. IEEE, vol. 88, no. 7, pp. 949-970, Jul. 2000.
[30] E. Semsar-Kazerooni and K. Khorasani, "Multi-agent team cooperation: A game theory approach," Automatica, vol. 45, no. 10, pp. 2205-2213, 2009.

[31] M. Nourian, P. Caines, R. Malhame, and M. Huang, "Nash, social and centralized solutions to consensus problems via mean field control theory," IEEE Trans. Automatic Control, vol. 58, no. 3, pp. 639-653, Mar. 2013.

[32] A. Yıldız and A. B. Özgüler, "Swarming behavior as Nash equilibrium," in Proc. NecSys 2012 Workshop, vol. 3, no. 1. pp. 151-155.

[33] A. C. Karmperis, K. Aravossis, I. P. Tatsiopoulos, and A. Sotirchos, "On the fair division of multiple stochastic pies to multiple agents within the Nash bargaining solution," PloS One, vol. 7, no. 9, p. e44535, 2012.

[34] D. E. Kirk, Optimal Control Theory: An Introduction. Mineola, NY, USA: Courier Dover Publications, 2012.

[35] N. N. Krasovskii, A. I. Subbotin, and S. Kotz, Game-Theoretical Control Problems. New York, NY, USA: Springer-Verlag, 1987.

[36] A. Bressan and W. Shen, "Small BV solutions of hyperbolic noncooperative differential games," SIAM J. Control Optimization, vol. 43, no. 1, pp. 194-215, 2004.

[37] B. Brandstatter and U. Baumgartner, "Particle swarm optimizationMass-spring system analogon," IEEE Trans. Magn., vol. 38, no. 2, pp. 997-1000, Mar. 2002.

[38] J. Buhl, D. Sumpter, I. Couzin, J. Hale, E. Despland, E. Miller, and S. Simpson, "From disorder to order in marching locusts," Science, vol. 312, no. 5778, pp. 1402-1406, 2006.

[39] A. Burger, "Arrival and departure behavior of common murres at colonies: Evidence for an information halo," Colonial Waterbirds, vol. 20, no. 1, pp. 55-65, 1997.

[40] L. E. Barnes, M. A. Fields, and K. P. Valavanis, "Swarm formation control utilizing elliptical surfaces and limiting functions," IEEE Trans. Syst., Man, Cybern. B, Cybern., vol. 39, no. 6, pp. 1434-1445, Dec. 2009.

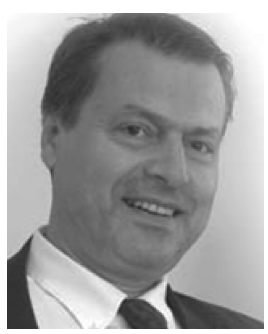

Arif Bülent Özgüler received the Ph.D. degree from the Electrical Engineering Department, University of Florida, Gainesville, FL, USA, in 1982.

He was a Researcher with the Marmara Research Institute of TÜBITAK, Kocaeli, Turkey, from 1983 to 1986. He spent one year at the Institut für Dynamische Systeme, Bremen Universität, Bremen, Germany, on Alexander von Humboldt Scholarship from 1994 to 1995 . He has been with the Department of Electrical and Electronics Engineering, Bilkent University, Ankara, Turkey, since 1986. He was with Bahçeşehir University, Istanbul, Turkey, in the 2008-2009 academic year, on leave from Bilkent University. He has published about 60 research papers in the field and is the author of two books, Linear Multichanne Control: A System Matrix Approach (Prentice Hall, 1994) and, with K. Saadaoui, Fixed Order Controller Design: A Parametric Approach (LAP Lambert Academic Publishing, 2010). His current research interests include decentralized control, stability robustness, realization theory, linear matrix equations, and applications of system theory to social sciences.

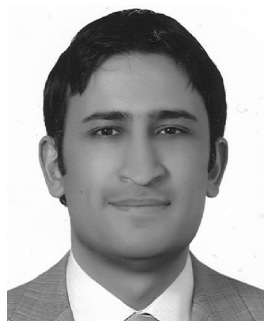

Aykut Yıldız (S'10) received the B.S. and M.S. degrees from the Electrical and Electronics Engineering Department, Bilkent University, Ankara, Turkey, in 2007 and 2010, respectively. He is currently pursuing the Ph.D. degree with the Department of Electrical and Electronics Engineering, Bilkent University.

From July 2007 to August 2011, he was a Research Assistant at the MLLDAR Project funded by the Scientific and Technical Research Council of Turkey (TÜBITAK). Since September 2011, he has been supported by the Servo Control Project by Military Electronics Industry Company (ASELSAN). Currently, he is a Teaching and Research Assistant with the Department of Electrical and Electronics Engineering, Bilkent University. His current research interests include control theory and swarm theory. 